\title{
Ferroptosis as a Novel Therapeutic Target for Friedreich's Ataxia ${ }^{\mathrm{S}}$
}

\author{
M. Grazia Cotticelli, Shujuan Xia, Daniel Lin, Taehee Lee, Leila Terrab, Peter Wipf, \\ Donna M. Huryn, and Robert B. Wilson
}

Department of Pathology and Laboratory Medicine, Children's Hospital Philadelphia, Philadelphia, Pennsylvania (M.G.C., S.X., D.L., T.L., R.B.W.); The Penn Medicine/CHOP Center of Excellence for Friedreich's Ataxia Research, Philadelphia, Pennsylvania (M.G.C., S.X., D.L., T.L., R.B.W.); Department of Chemistry, University of Pittsburgh, Pittsburgh, Pennsylvania (L.T., P.W.); Department of Chemistry (D.M.H.), and Perelman School of Medicine (R.B.W.), University of Pennsylvania, Philadelphia, Pennsylvania

Received August 8, 2018; accepted January 4, 2019

\begin{abstract}
Friedreich ataxia (FRDA) is a progressive neuro- and cardiodegenerative disorder characterized by ataxia, sensory loss, and hypertrophic cardiomyopathy. In most cases, the disorder is caused by GAA repeat expansions in the first introns of both alleles of the FXN gene, resulting in decreased expression of the encoded protein, frataxin. Frataxin localizes to the mitochondrial matrix and is required for iron-sulfur-cluster biosynthesis. Decreased expression of frataxin is associated with mitochondrial dysfunction, mitochondrial iron accumulation, and increased oxidative stress. Ferropotosis is a recently identified pathway of regulated, iron-dependent cell death, which is biochemically distinct from apoptosis. We evaluated whether there is evidence for ferroptotic pathway activation in cellular models of FRDA. We found that primary patient-derived fibroblasts, murine fibroblasts with FRDA-associated mutations, and murine fibroblasts in which a repeat expansion had been
\end{abstract}

introduced (knockin/knockout) were more sensitive than normal control cells to erastin, a known ferroptosis inducer. We also found that the ferroptosis inhibitors ethyl 3-(benzylamino)4-(cyclohexylamino)benzoate (SRS11-92) and ethyl 3-amino4-(cyclohexylamino)benzoate, used at $500 \mathrm{nM}$, were efficacious in protecting human and mouse cellular models of FRDA treated with ferric ammonium citrate (FAC) and an inhibitor of glutathione synthesis [L-buthionine $(S, R)$-sulfoximine (BSO)], whereas caspase-3 inhibitors failed to show significant biologic activity. Cells treated with FAC and BSO consistently showed decreased glutathione-dependent peroxidase activity and increased lipid peroxidation, both hallmarks of ferroptosis. Finally, the ferroptosis inhibitor SRS11-92 decreased the cell death associated with frataxin knockdown in healthy human fibroblasts. Taken together, these data suggest that ferroptosis inhibitors may have therapeutic potential in FRDA.

\section{Introduction}

Friedreich ataxia (FRDA) is the most common inherited ataxia with a birth incidence of approximately 1 in 40,000. FRDA is characterized by progressive degeneration of large sensory neurons in the dorsal root ganglia, which causes ataxia, dysarthria, areflexia, and sensory loss (Collins, 2013; Gomes and Santos, 2013; Koeppen and Mazurkiewicz, 2013). Although FRDA is a neurologic disorder, early mortality is almost exclusively due to hypertrophic cardiomyopathy, which develops in a majority of patients. FRDA is caused by decreased expression of the protein frataxin, in most cases

This work was supported by the Friedreich's Ataxia Research Alliance, and the Hamilton and Finneran families.

https://doi.org/10.1124/jpet.118.252759.

S This article has supplemental material available at jpet.aspetjournals.org. secondary to a GAA repeat expansion in the first intron of both alleles of the FXN gene. Disease-associated missense mutations have also been described, many of which lead to protein instability and decreased frataxin levels. Frataxin protein localizes in the mitochondria where it is involved in the biosynthesis of iron-sulfur clusters, which are prosthetic groups important for the function of many proteins, including critical subunits of the mitochondrial electron transport chain. At the cellular level, there is evidence of mitochondrial iron accumulation (Delatycki et al., 1999; Wong et al., 1999; Calmels et al., 2009) as well as mitochondrial dysfunction (Rötig et al., 1997; Bradley et al., 2000; Lodi et al., 2001). Chronic oxidative stress in FRDA cells (Armstrong et al., 2010), and more recently lipid peroxidation (Cotticelli et al., 2013), have been implicated in the pathophysiology of the disorder. Currently, there is no cure or Food and Drug Administration-approved treatment available for FRDA patients.

ABBREVIATIONS: BSO, L-buthionine ( $S, R$ )-sulfoximine; FAC, ferric ammonium citrate; Fer-1, ethyl 3-amino-4-(cyclohexylamino)benzoate; FRDA, Friedreich ataxia; GPX, phospholipid hydroperoxide glutathione peroxidase; KIKO, knockin/knockout; KIWT, knockin/wild type; OD, optical density; siRNA, small interfering RNA; SRS11-92, ethyl 3-(benzylamino)-4-(cyclohexylamino)benzoate; XJB-5-131 (1-[(2S,3E,5S)-5[[(1,1-Dimethylethoxy)carbonyl]amino]-7-methyl-1-oxo-2-(phenylmethyl)-3-octen-1-yl]-L-prolyl-L-valyl-N5-[(phenylmethoxy)carbonyl]-N(2,2,6,6-tetramethyl-1-oxy-4-piperidinyl)-L-ornithinamide); Z-DEVD-FMK, carbobenzoxy-aspartyl-glutamyl-valyl-aspartyl-[O-methyl]-fluoromethylketone; Z-VAD-FMK, carbobenzoxy-valyl-alanyl-aspartyl-[O-methyl]-fluoromethylketone. 
Ferroptosis was first described (Dixon et al., 2012) as a necrotic cell death pathway, triggered by iron accumulation and lipid peroxidation, which causes morphologic and biochemical changes distinct from apoptosis and other necrotic pathways. The details of the process are still under investigation, but it is known that during the last stage of the process the direct or indirect inactivation of phospholipid hydroperoxide glutathione peroxidase (GPX) 4 causes accumulation of peroxidized polyunsaturated fatty acids, which ultimately leads to cell death (Yang et al., 2016). Ferroptosis has been implicated in neurodegeneration, and ferroptosis inhibitors have been tested in models of neurodegenerative disorders, including Parkinson's and Alzheimer's disease (Stockwell et al., 2017). Because key biochemical defects in FRDA cellsmitochondrial iron accumulation, increased oxidative stress, and lipid peroxidation-are essentially the hallmarks of ferroptosis, we explored the possibility that ferroptosis might play a role in FRDA by testing the sensitivity of FRDA cells to a known ferroptosis inducer, and we analyzed the effects of known antiferroptotic agents in cellular models of FRDA.

\section{Materials and Methods}

Cells. The murine FRDA cell line, I154F (Frda ${ }^{\mathrm{L} 3 / \mathrm{L}-;}$ h-I54F) , is a fibroblast cell line in which one allele of the murine frataxin gene is knocked out and the other allele is replaced by the human frataxin gene with the disease-associated missense mutation, I154F (Calmels et al., 2009). The control cell line, $2 \mathrm{~F} 1$, is a murine fibroblast line in which one allele of the murine frataxin gene is knocked out and the other allele is replaced by the normal human frataxin gene. The generation of these lines and their biochemical properties have been previously described (Calmels et al., 2009).

Lung tissues from wild-type, knockin/knockout (KIKO), and knockin/wild-type (KIWT) mice (Miranda et al., 2002) were kindly provided by Dr. David Lynch (Children's Hospital of Philadelphia). KIKO mice, in which a $(\mathrm{GAA})_{230}$ repeat expansion was knocked in on one frataxin allele and the other frataxin allele was knocked out, and KIWT mice, in which a $(\mathrm{GAA})_{230}$ repeat expansion was knocked in on one frataxin allele and the other frataxin allele was wild type, have been previously described (Miranda et al., 2002). Fibroblasts were isolated and cultured from mouse lung tissues as previously described by Seluanov et al. (2010), with Liberase obtained from Roche. Frataxin expression was confirmed by ELISA (Abcam, Cambridge, MA).

We obtained primary human FRDA fibroblasts (GM3816 and GM4078) and healthy control fibroblasts (GM8399) from Coriell (Coriell Institute for Medical Research, Camden, NJ). The FRDA fibroblasts have homozygous GAA repeat expansions in the first intron of the FRDA gene: both have moderate GAA repeat expansions of 223 and 490 repeats (GM3816) or 357 and 523 repeats (GM4078). Normal primary human fibroblasts (DL6030) were derived from the practice of Dr. David Lynch at the Children's Hospital of Philadelphia. Subjects were recruited using the Friedreich's Ataxia Research Alliance database, by listing on ClinicalTrials.gov (NCT01965327; https://clinicaltrials.gov/), and through the practice of Dr. Lynch. The Declaration of Helsinki protocols were followed and all subjects provided written, informed consent prior to participation. The Children's Hospital of Philadelphia Institutional Review Board approved the study. Subjects were provided with a modest stipend for each visit.

Cells were grown in Dulbecco's modified Eagle's medium with low glucose (Life Technology, Carlsbad, CA) supplemented with $10 \%$ fetal bovine serum (Hyclone, Pittsburgh, PA) and 1\% penicillin/streptomycin (Life Technology).

Erastin Treatment. For both murine and human fibroblasts, 10,000 cells/well were seeded into 48 -well microtiter plates. The following day, erastin or drug vehicle was added (at concentrations indicated in the text and figures). The final concentration of drug vehicle (DMSO) was $0.1 \%$. Then, 24 or 48 hours later, the cells were washed with PBS and viability was assessed by chemiluminescence measurement of intracellular ATP using the CellTiter-Glo assay kit (Promega, Madison, WI) as per the manufacturer's instructions. Erastin (purity 99.27\%) was purchased from Selleckchem (Houston, TX).

Ferric Ammonium Citrate Plus L-Buthionine $(S, R)$-Sulfoximine Treatment. Both murine and human FRDA cells show increased sensitivity to treatment with ferric ammonium citrate (FAC), iron content $16.5 \%-18.5 \%$ (Sigma, St. Louis, MO), and L-buthionine $(S, R)$ sulfoximine (BSO), 99\% purity (Acros Organics, West Chester, PA), which inhibits the rate-limiting step of glutathione synthesis. Under the assay conditions we use, concentrations of iron and BSO that by themselves are nontoxic together cause a synergistic loss of viability (Cotticelli et al., 2012). Drugs are typically added 2 hours after BSO treatment. The assay has been described in detail and validated multiple times (Cotticelli et al., 2012, 2013). Idebenone (purity $\geq 98 \%$ ) was obtained from Spectrum Chemical (New Brunswick, NJ); carbobenzoxy-valylalanyl-aspartyl-[O-methyl]-fluoromethylketone (purity $\geq 95 \%$ ) was obtained from Adipogen (San Diego, CA); staurosporine (purity $\geq 98 \%$ ) and Z-DEVD-FMK (purity $\geq 95 \%$ ) (carbobenzoxy-aspartyl-glutamylvalyl-aspartyl-[O-methyl]-fluoromethylketone) were obtained from Cayman Chemicals (Ann Arbor, MI).

Caspase 3 Measurements. Murine fibroblasts were seeded into 48-well microtiter plates at 12,000 cells/well and FAC was added to a final concentration of $20 \mu \mathrm{g} / \mathrm{ml}$ (day 0 ). The following day (day 1), BSO was added to a final concentration of $50 \mu \mathrm{M}$. Caspase 3 and viability were assessed in parallel wells by chemiluminescence measurements using Caspase-Glo 3/7 and CellTiter-Glo assay kits (Promega), respectively, as per the manufacturer's instructions. Measurements were done on day 2 or 3 . Activation of caspase 3 in the samples was compared with caspase 3 activation induced by overnight treatment (16 hours) with staurosporine (purity $\geq 98 \%$; Cayman Chemical) at a final concentration of $10 \mu \mathrm{M}$.

Chemiluminescence Measurements. Chemiluminescence measurements were performed using a Synergy $\mathrm{H} 1$ microplate reader (Biotek, Winooski, VT). When the assay was performed in 48-well plates, the cells were lysed and a fraction of the lysate was transferred to opaque white plates by using adjustable multichannels pipets.

Lipid Peroxidation Measurements. To measure lipid peroxidation in the FAC/BSO model, murine (10,000 cells/well) or human (4500 cell/well) fibroblasts were seeded in black-wall, clear-bottom 96-well plates and treated with FAC and BSO as described previously. On the day of measurement, Bodipy 581/591 C11 (Invitrogen, Eugene, OR) was added at $10 \mu \mathrm{M}$ final concentration. Following 1-hour incubation at $37^{\circ} \mathrm{C}$, cells were washed three times with PBS and fluorescence was read at $488_{\text {excitation }} / 510_{\text {emission }}$ and $581_{\text {excitation }} / 591_{\text {emission }}$ wavelengths. The fluorescence ratio $591_{\text {[reduction] }} / 510_{\text {[oxidation] }}$ was calculated and normalized by cell viability assessed in parallel plates. Lipid peroxidation in the samples was compared with lipid peroxidation induced in the human or murine fibroblasts by overnight treatment with $100 \mathrm{nM}$ RSL3 or 2 hours treatment with $1 \mu \mathrm{M}$ RSL3 (purity 99.76\%; Selleckchem), respectively. Fluorescence measurements were performed using a monochromator-equipped Synergy $\mathrm{H} 1$ microplate reader (Biotek).

Glutathione-Dependent Peroxidase Assay. GPX activity was measured using the Glutathione Peroxidase Assay kit (Abcam) as per the manufacture's instructions. The enzymatic reaction was run in UV-transparent, 96-well plates (Corning, Kennebunk, ME) and $\mathrm{NADPH}$ oxidation was monitored by optical density (OD) at $340 \mathrm{~nm}$ over 20 minutes at $25^{\circ} \mathrm{C}$ in a Synergy $\mathrm{H} 1$ microplate reader (Biotek).

Frataxin Knockdown Model. The frataxin knockdown model has been described previously (Cotticelli et al., 2018). Briefly, normal primary human fibroblasts (DL6030) were seeded in $100 \mathrm{~mm}$ dishes at 70,000 cells/dish. Three days later (day 1 ), the cells were transfected overnight using double-strand small interfering RNA (siRNA) against frataxin mRNA (Origene, Rockville, MD) or a scrambled siRNA as control. The following day (day 2), the medium was exchanged. In the 
present study, ethyl 3-(benzylamino)-4-(cyclohexylamino)benzoate (SRS11-92), Z-DEVD-FMK, or carrier control was added on days 2 and 4. Cells were counted on day 5 using the Countess Cell Counter (Invitrogen, Carlsbad, CA). Double stranded siRNAs were transfected using RNAimax reagent (Life Technology) at a final concentration of $10 \mathrm{nM}$ as per the manufacturer's instructions. To measure caspase 3 activation in the frataxin knockdown model, 2000 cells were retrotransfected in white opaque 96-well plates on day 1 and transfected again on day 3 . Staurosporine was added at $10 \mu \mathrm{M}$ overnight on days 2 and 4 . Measurements were obtained on days 3 and 5 as described previously. To measure lipid peroxidation in the frataxin knockdown model, 2000 cells were retrotransfected in black-wall, clear-bottom 96-well plates on day 1 and transfected again on day 3. RSL3 was added at $500 \mathrm{nM}$ overnight on day 2 or 4 and measurements were obtained on days 3 and 5 , as described previously.

Chemical Compounds. Compound structures are shown in Supplemental Fig. 1. Ethyl 3-amino-4-(cyclohexylamino)benzoate (Fer-1) was synthesized according to published procedures (Skouta et al., 2014). XJB-5-131 (1-[(2S,3E,5S)-5-[[(1,1-Dimethylethoxy)carbonyl]amino]7-methyl-1-oxo-2-(phenylmethyl)-3-octen-1-yl]-L-prolyl-L-valyl-N5[(phenylmethoxy)carbonyl]-N-(2,2,6,6-tetramethyl-1-oxy-4-piperidinyl)L-ornithinamide) was synthesized as described previously (Wipf et al., 2005). The purity of each was $>95 \%$ as determined by liquid chromatography/high-resolution mass spectrometry analysis. SRS11-92 was prepared as follows: a solution of Fer-1 $(0.380 \mathrm{~g}, 1.45 \mathrm{mmol})$ and tetrabutylammonium bromide $(2.58 \mathrm{~g}, 8.00 \mathrm{mmol})$ in acetonitrile $(16 \mathrm{ml})$ was treated with benzyl chloride $(0.437 \mathrm{ml}, 3.78 \mathrm{mmol})$ in three portions over a period of 8 hours at room temperature. The mixture was treated with water $(30 \mathrm{ml})$ and the aqueous layer was extracted with $\mathrm{CH}_{2} \mathrm{Cl}_{2}$ $(2 \times 30 \mathrm{ml})$. The organic layers were combined, washed with brine, dried $\left(\mathrm{Na}_{2} \mathrm{SO}_{4}\right)$, filtered, and concentrated. Purification by chromatography on $\mathrm{SiO}_{2}$ (3\%-20\% EtOAc in hexanes) yielded SRS11-92 (0.141 g, 28\%) as a viscous brown oil: Infrared Spectroscopy (IR) $\left(\mathrm{CH}_{2} \mathrm{Cl}_{2}\right) 3396,2929$, $2853,1701,1681,1598,1525$, and $1283 \mathrm{~cm}^{-1}$; ${ }^{1} \mathrm{H}$ NMR $(400 \mathrm{MHz}$; $\left.\mathrm{DMSO}_{-} d_{6}\right) \delta 7.38-7.31(\mathrm{~m}, 4 \mathrm{H}), 7.26-7.21(\mathrm{~m}, 2 \mathrm{H}), 6.94(\mathrm{~d}, J=1.9 \mathrm{~Hz}$, $1 \mathrm{H}), 6.49(\mathrm{~d}, J=8.6 \mathrm{~Hz}, 1 \mathrm{H}), 5.50(\mathrm{t}, J=5.8 \mathrm{~Hz}, 1 \mathrm{H}), 5.18(\mathrm{~d}, J=7.3 \mathrm{~Hz}$, $1 \mathrm{H}), 4.30(\mathrm{~d}, J=5.4 \mathrm{~Hz}, 2 \mathrm{H}), 4.13(\mathrm{q}, J=7.1 \mathrm{~Hz}, 2 \mathrm{H}), 3.37-3.28(\mathrm{~m}, 1 \mathrm{H})$, 2.01-1.96 (m, $2 \mathrm{H}), 1.77-1.71$ (m, $2 \mathrm{H}), 1.65-1.60$ (m, $1 \mathrm{H}), 1.42-1.31$ $(\mathrm{m}, 2 \mathrm{H})$, and $1.27-1.16(\mathrm{~m}, 6 \mathrm{H}) ;{ }^{13} \mathrm{C} \mathrm{NMR}\left(126 \mathrm{MHz}\right.$; DMSO- $\left.d_{6}\right)$ $\delta 166.3,139.8,139.7,134.2,128.2,127.3,126.7,120.6,116.5,110.4$, $107.7,59.2,50.8,47.1,32.5,25.5,24.6$, and 14.3; high-resolution mass spectrometry $\left(\mathrm{ESI}^{+}\right.$) mass-to-charge value calculated for $\mathrm{C}_{22} \mathrm{H}_{29} \mathrm{O}_{2} \mathrm{~N}_{2}$ $[\mathrm{M}+\mathrm{H}]$ 353.2224, found 353.2222; and Evaporating Light Scattering Detection (ELSD) purity $100 \%$.

Statistical Analyses. The $\mathrm{EC}_{50}$ value was calculated using log (inhibitor) versus response-variable slope (four parameters) from GraphPad (GraphPad Software, Inc.). Unless otherwise indicated, the $P$ values were derived from Student's $t$ test and are two sided. Independent replicates within an experiment refer to cells that were plated independently, cultured independently, treated independently (transfected, combined with drug or carrier control), and analyzed independently. Where indicated, we repeated experiments in their entirety to confirm results. Because primary cells change with passage number, and because infection and transfection efficiencies differ from one experiment to the next, we generally did not combine data from confirmatory independent experiments.

\section{Results}

FRDA Cells Are Hypersensitive to the Ferroptosis Inducer Erastin. Erastin is a small molecule inhibitor of the cystine-glutamate antiporter that was first identified as being selectively toxic in a cell line expressing an oncogenic Ras mutation and was later identified as an inducer of ferroptosis (Dixon et al., 2012). We treated primary human fibroblasts (GM3816) derived from a patient with FRDA, and normal age- and gender-matched primary human fibroblasts
(GM8399), with increasing doses of erastin for 24 and 48 hours. Treatment with erastin caused cell death in a dose-dependent manner. The 48-hour treatments with 5 or $10 \mu \mathrm{M}$ erastin resulted in survival of $55 \%$ and $34 \%$ of the control cells, respectively, whereas only $10 \%$ and $4 \%$ of FRDA cells survived, respectively (Fig. 1A). The difference in sensitivity was most evident after 24 hours of treatment with $5 \mu \mathrm{M}$ erastin: 89\% of the control cells survived, while only $16 \%$ of the FRDA cells survived (Fig. 1A).

Similar results were obtained using I154F murine fibroblasts, a missense-mutation cellular model of FRDA, and the corresponding normal control cells, $2 \mathrm{~F} 1$. Compared with human fibroblasts, both the $\mathrm{I} 154 \mathrm{~F}$ and $2 \mathrm{~F} 1$ cells were more sensitive to erastin, since 24-hour treatment resulted in a large loss of viability in both. However, the survival of the control cells was 2-fold higher than that of the FRDA cells when the cells were treated with $1 \mu \mathrm{M}$ erastin, and 4- and 5-fold higher when the cells were treated with 10 and $5 \mu \mathrm{M}$ erastin, respectively (Fig. 1B).

Finally, we tested whether primary fibroblasts derived from the KIKO mouse model of FRDA were also hypersensitive to erastin. KIKO mice, in which a repeat expansion of 230 GAAs was knocked in to one frataxin allele while the second allele was knocked out, are widely used as a mouse model of FRDA (Miranda et al., 2002; Lin et al., 2017). Lung fibroblasts derived from KIKO mice, as well as from control KIWT and wild-type mice, were established (see Materials and Methods) and frataxin levels in the cultured KIKO cells were 30\% compared with KIWT and wild-type cells (Supplemental Fig. 2). As shown in Fig. 1C, 24-hour treatment with $1 \mu \mathrm{M}$ erastin resulted in almost complete (97\%) cell death for the KIKO cells, whereas $25 \%$ of control cells survived. The results using $10 \mu \mathrm{M}$ erastin were similar, with $99 \%$ cell death for KIKO cells and $27 \%$ survival for control cells (Fig. 1C).

Ferroptosis Inhibitors Protect FRDA Cells from Iron and a Glutathione-Synthesis Inhibitor. We tested the efficacy of the well-established ferroptosis inhibitors Fer-1 and SRS11-92 (Skouta et al., 2014) (Supplemental Fig. 1) at concentrations ranging from $50 \mu \mathrm{M}$ to $5 \mathrm{nM}$ in the murine I154F FRDA fibroblasts. These cells are sensitive to combined treatment with iron, in the soluble form of FAC, and BSO (which inhibits glutathione synthesis) at concentrations that by themselves are nontoxic but together cause a synergistic loss of viability (Cotticelli et al., 2012). Under the assay conditions, $75 \%$ of the cells died 48 hours following treatment (Fig. 2A), and treatment with $1 \mu \mathrm{M}$ idebenone, used as a positive control, resulted in complete rescue (Fig. 2A). Both Fer-1 and SRS11-92 rescued the cells completely at $500 \mathrm{nM}$ (Fig. 2A). The efficacy of these compounds was consistently high between $50 \mu \mathrm{M}$ and $500 \mathrm{nM}$. Of note, treatment of murine and human fibroblasts with either drug up to $50 \mu \mathrm{M}$ resulted in no toxicity (data not shown). Using the same assay, we also tested XJB-5-131 (Wipf et al., 2005) (Supplemental Fig. 1), a mitochondrially targeted antiferroptotic and antiapoptotic compound. XJB-5-131 was efficacious at $1 \mu \mathrm{M}$, but showed some toxicity at $10 \mu \mathrm{M}$ (Fig. 2B), most likely due to its high level of enrichment in mitochondrial membranes. In a parallel assay, the caspase-3 inhibitor Z-DEVDFMK showed a small, statistically significant positive effect, but only when used at $50 \mu \mathrm{M}$ (data not shown). In a typical assay, drugs were administered 2 hours after BSO treatment. To test whether the time of drug administration was important, we repeated the experiment using SRS11-92 and Z-DEVD-FMK 

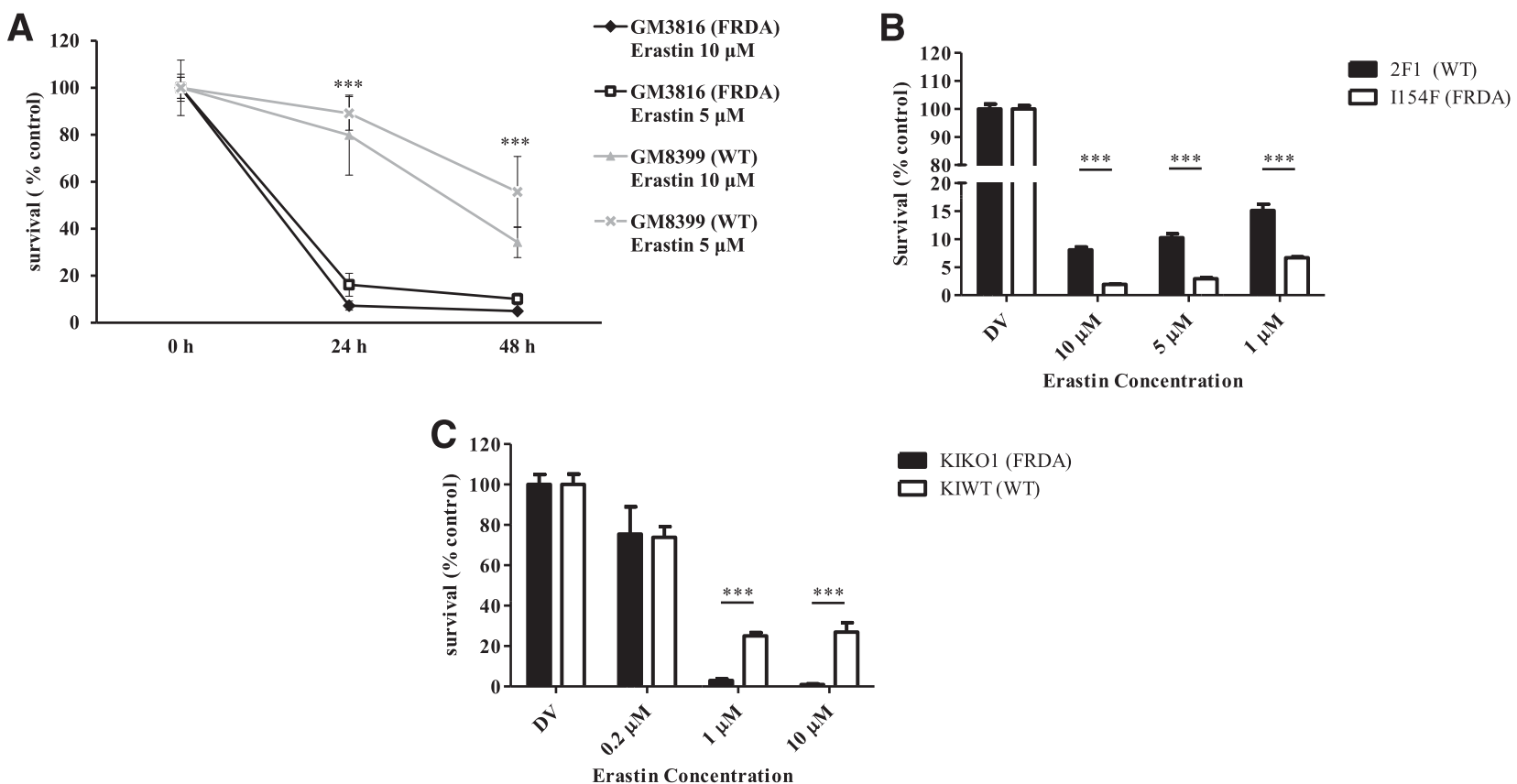
$\operatorname{KIKO1}(\mathrm{FRDA})$
$\operatorname{KIWT}(\mathrm{WT})$

Fig. 1. Friedreich ataxia cells are hypersensitive to the ferroptosis inducer erastin. (A) Primary human FRDA fibroblasts (3816) and primary human normal-control fibroblasts (GM8399) were seeded in 48-well plates and treated with 5 or $10 \mu \mathrm{M}$ erastin. Cell viability was measured after 24 or 48 hours by chemiluminescence measurement of intracellular ATP. (B) Murine I154F fibroblasts, a missense-mutation model of Friedreich ataxia, were seeded in 48-well plates and treated with 1, 5, or $10 \mu \mathrm{M}$ erastin. Cell viability was measured after 24 hours by chemiluminescence measurement of intracellular ATP. (C) Primary fibroblasts derived from KIKO (FRDA) or KIWT (heterozygous wild-type) mice were seeded in 48-well plates and treated with 0.2 , 1 , or $10 \mu \mathrm{M}$ erastin. Cell viability was measured after 24 hours by chemiluminescence measurement of intracellular ATP. The experiments shown are representative of at least two independent experiments, and the data shown are the mean and S.D. values calculated from four replicates. $* * * P<0.001$ by two-sided $t$ test.

administered either 2 or 24 hours after BSO treatment. Figure 3A shows that the ferroptosis inhibitor, which was highly efficacious when administered at early time points, was inactive when administered at a later time point. In contrast, Z-DEVD-FMK was only slightly efficacious when used at $20 \mu \mathrm{M}$ at an early time point but could rescue up to $15 \%$ of cells when administered at the later time point (Fig. 3A). The pan-caspase inhibitor carbobenzoxy-valyl-alanylaspartyl-[O-methyl]- fluoromethylketone had no effect at either time point when used at $20 \mu \mathrm{M}$ (Fig. 3A). To further explore the role of caspase 3 in the assay, we measured caspase 3 activity in I154F cells treated with FAC and BSO at 24 and 48 hours following BSO treatment. Caspase 3 activity was compared with the activity measured in untreated cells (basal level/negative control) and cells treated overnight with $10 \mu \mathrm{M}$ staurosporine (100\% activation/positive control) (Belmokhtar et al., 2001). After 24 hours of exposure to FAC and BSO, caspase 3 activity increased 14-fold, whereas overnight treatment with staurosporine increased caspase 3 activity up to 46-fold; after 48 hours of FAC and BSO treatment,
A

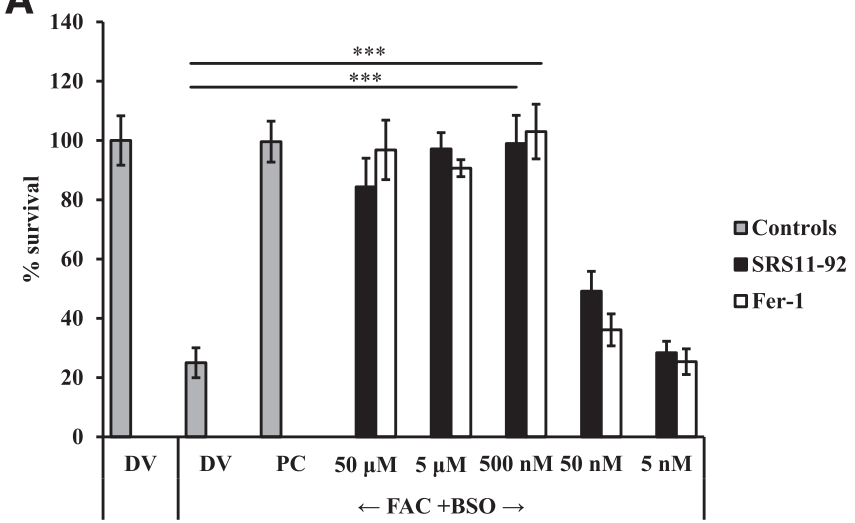

B

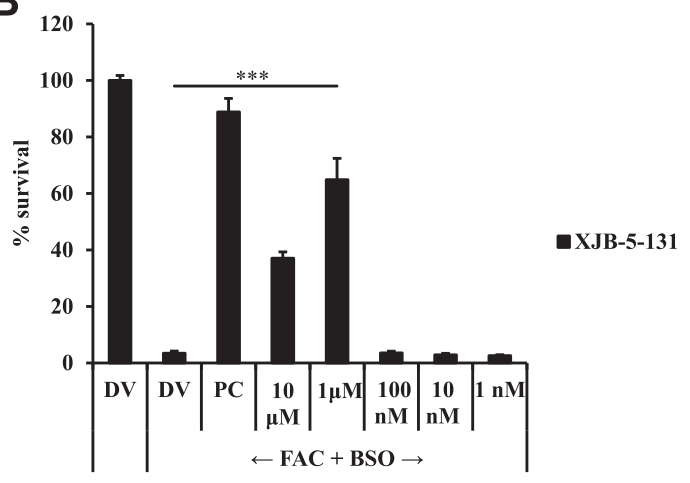

Fig. 2. Ferroptosis inhibitors rescue murine I154F fibroblasts treated with iron and BSO in a dose-dependent manner. Murine I154F fibroblasts, a missense-mutation model of Friedreich ataxia, were seeded in 48-well plates and treated with a combination of FAC and BSO for 48 hours. Drugs Fer- 1 or SRS11-92 (A) or XJB-5-131 (B), were added 2 hours after BSO addition; viability was assessed by chemiluminescence measurement of intracellular ATP (see Materials and Methods). Idebenone, used as a positive control (PC), was tested at $1 \mu \mathrm{M}$. The final concentration of the drug vehicle (DV), DMSO, was $0.5 \%$. The experiments are representative of at least two independent experiments, and the data shown are the mean and the S.D. values calculated from six replicates. ${ }^{* * *} P<0.001$ by two-sided $t$ test. 


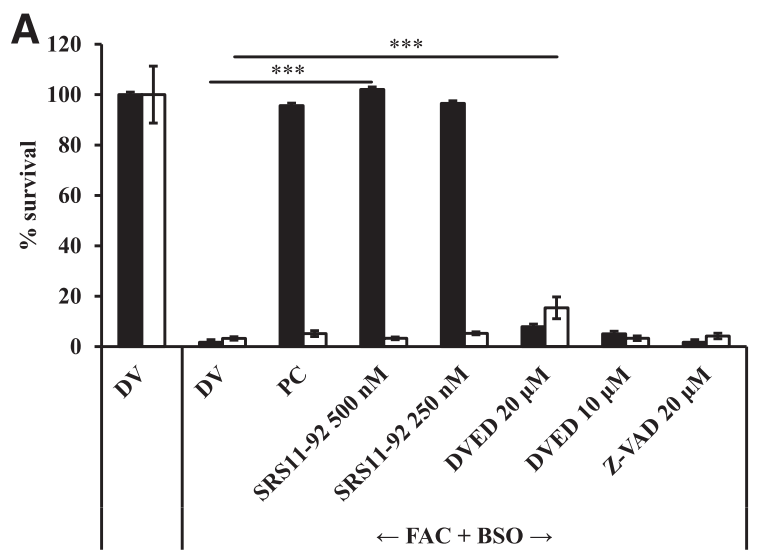

B
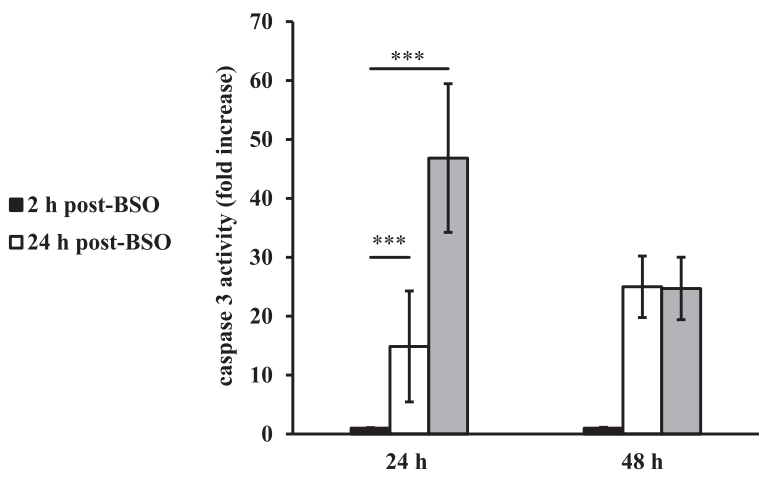

DDV

口FAC+BSO

口STS $10 \mathrm{uM}$

Fig. 3. Caspase 3 activation in murine I154F fibroblasts treated with iron and BSO. (A) Murine I154F fibroblasts, a missense-mutation model of Friedreich ataxia, were seeded in 48-well plates and treated with a combination of FAC and BSO for 48 hours. One micromolar idebenone of the positive control, the ferroptosis inhibitor SRS11-92, the caspase 3-7 inhibitor Z-DVED-FMK, or the pan-caspase inhibitor Z-VAD-FMK was added at the concentrations indicated, either 2 or 24 hours after BSO addition. Viability was assessed by chemiluminescence measurement of intracellular ATP. The final concentration of the drug vehicle (DV), DMSO, was $0.5 \%$. The experiments are representative of at least two independent experiments, and the data shown are the mean and S.D. values calculated from six replicates. $* * * P<0.001$ by two-sided $t$ test. (B) Murine I154F fibroblasts were seeded in 48 -well plates and treated with a combination of FAC and BSO for 48 hours. Caspase 3 activation was measured after 24 or 48 hours following BSO treatment. As a positive control for caspase 3 activation, cells seeded in parallel were treated with $10 \mu \mathrm{M}$ staurosporine (STS) overnight; as a negative control, cells were treated with DV, DMSO. Caspase 3 activation was assessed by chemiluminescence measurement (see Materials and Methods) and normalized by cell number. The experiments are representative of at least two independent experiments, and the data shown are the mean and S.D. values calculated from six replicates. ${ }^{* * *} P<0.001$ by two-sided $t$ test.

caspase 3 activity was as high as in cells treated overnight with staurosporine (Fig. 3B). Finally, we tested Fer-1 and SRS11-92 in primary, patient-derived fibroblasts (GM4078) treated with FAC and BSO. We found that the results were consistent with the data obtained using the mouse cells: the $\mathrm{EC}_{50}$ values were $12 \mathrm{nM}$ for Fer-1 (Hill slope 0.4553 $R^{2}=0.9596$ ) and $58 \mathrm{nM}$ for SRS11-92 (Hill slope 0.3679 $R^{2}=0.9347$; Supplemental Fig. 3).

Activation of the Ferroptosis Pathway in the FAC/BSO Model. To confirm the activation of the ferroptosis pathway in the FAC/BSO model, we treated primary human FRDA fibroblasts with FAC at $150 \mu \mathrm{g} / \mathrm{ml}$ and BSO at $100 \mu \mathrm{M}$ as described previously and measured glutathione-dependent peroxidase (GPX) activities (Fig. 4A) and lipid peroxidation on day 4, 48 hours after BSO addition. The GPX enzymatic activity in the cells treated with FAC and BSO decreased by $70 \%$ in treated cells compared with controls (Fig. 4B). At the same time, lipid peroxidation went up slightly on day 3 (data not shown) but tripled on day 4 (Fig. 4C). This increase was comparable to the level of lipid peroxidation achieved by overnight treatment with RSL3, a glutathione-dependent peroxidase 4 inhibitor and known ferroptosis inducer (Fig. 4C). Similar results were seen in the murine fibroblasts $\mathrm{I} 154 \mathrm{~F}$, where lipid peroxidation on day 3 increased dramatically and GPX activity decreased by $30 \%$ compared with control cells (data not shown).

Ferroptosis Inhibitors Rescue Death Induced by Frataxin Knockdown. We described previously a cellular model of FRDA obtained by knocking down frataxin mRNA using siRNA in normal primary human fibroblasts: transfecting the cells with an antifrataxin siRNA results in loss of $98 \%$ of frataxin mRNA and loss of 50\% of frataxin protein at 48 hours post-transfection; when the cells are transfected a second time, frataxin protein levels are close to $10 \%$ on day 7 after the first transfection, but after that point the cells are dying in large numbers and no further analyses are possible (Cotticelli et al., 2018). To test whether SRS11-92 could prevent cell death induced by frataxin knockdown, we transfected the cells with
anti-FXN siRNA or with a scrambled siRNA and then treated the cells every 48 hours (twice total, on days 2 and 4) with the drug at $500 \mathrm{nM}$, or with drug vehicle. On day 5 after transfection, we counted the cells. The results (Fig. 5A) show that $43 \%$ of the cells in which frataxin was knocked down were viable, compared with cells in which a scrambled construct was transfected, whereas $77 \%$ of the cells treated with SRS1192 survived. Compound treatment was continued on days 6 , 9 , and 11 , while transfecting the cells a second time on day 8 resulted in $85 \%$ cell death, at which point the drug did not have any effect (day 12, data not shown). To better understand the mechanism by which cells die after frataxin levels begin to decrease ( $43 \%$ cell death as shown in Fig. 5A), we focused on detecting at what point lipid peroxidation and caspase 3 activity increased. Surprisingly, we could not detect any difference in lipid peroxidation or caspase 3 activity on day 4 post-transfection in DL6030 compared with control cells. Repeating the experiment in the GM8399 cell line confirmed these results (data not shown). However, in both cells lines, DL6030 and GM8399, frataxin knockdown increased the lipid peroxidation induced by the GPX-4 inhibitor RSL3, but did not increase caspase 3 activity induced by staurosporine 48 hours after the first transfection (Fig. 5, B and C, and data not shown). Figure 5B (left) shows that in the presence of RSL3, lipid peroxidation in DL6030 cells in which frataxin was knocked down is already 1.4 -fold higher than in controltransfected cells 48 hours after the first transfection, and more than 7-fold higher after a second transfection (Fig. 5B, right). In comparison, increased sensitivity to staurosporine was unchanged at 48 hours (Fig. 5C, left) and could be detected only when the cells were transfected a second time (Fig. 5C, right).

\section{Discussion}

The aim of this study was to determine whether there is evidence of activation of the ferroptosis pathway in FRDA. 

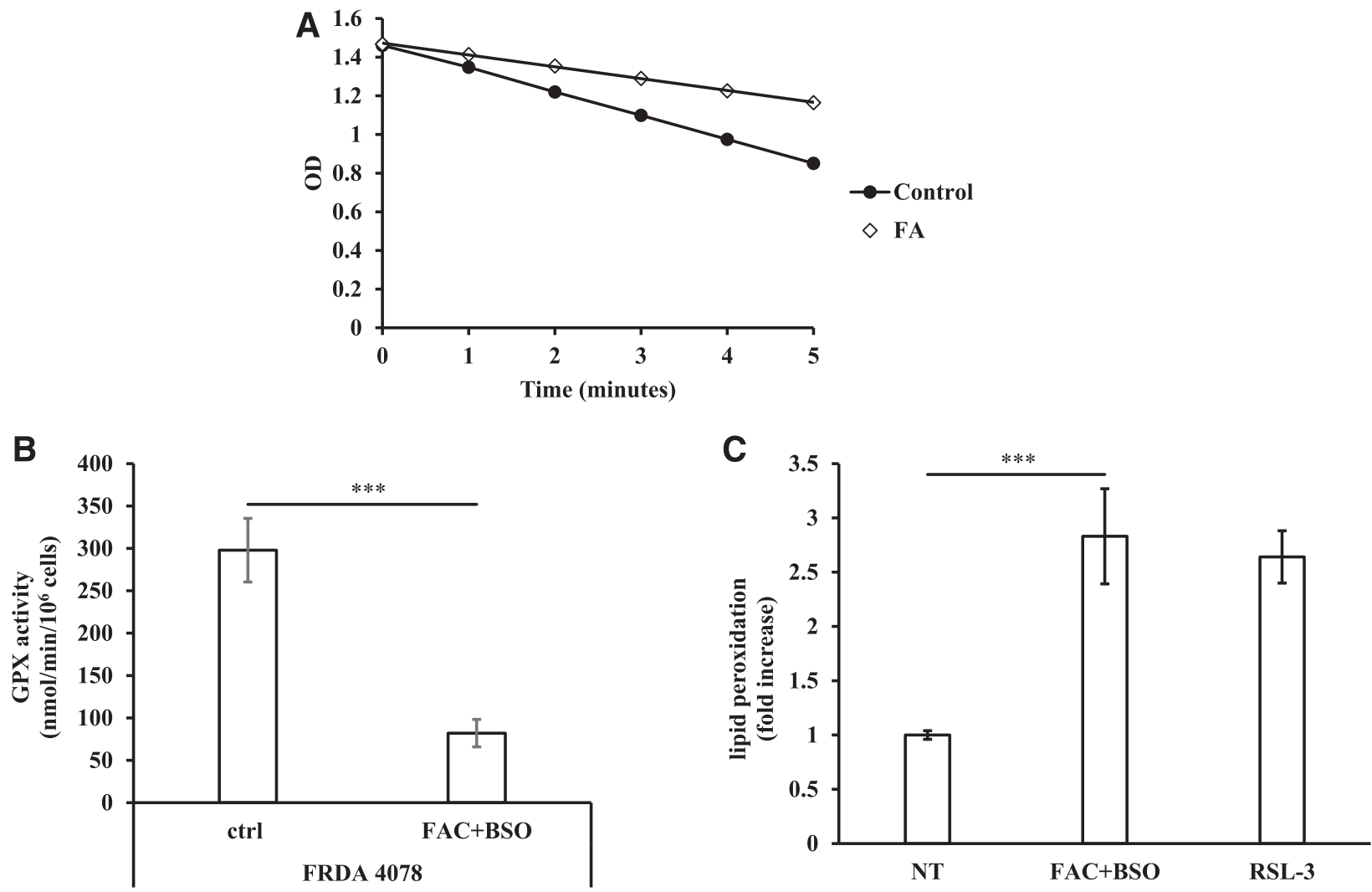

Fig. 4. Activation of ferroptosis in cells treated with iron and BSO. Primary human fibroblasts GM4078 were seeded in dishes and treated with a combination of FAC and BSO as described in Materials and Methods. On day 4, 48 hours post BSO addition, the cells were lysed and enzymatic activity was measured. (A) Graphic representation of GPX enzymatic assay: glutathione-dependent peroxidase (GPX) activity was measured following OD at $340 \mathrm{~nm}$ for 20 minutes at $25^{\circ} \mathrm{C}$. (B) GPX enzymatic activity (nanomoles per minute per million cells) of FRDA GM4078 not treated or treated with FAC and BSO. (C) To measure lipid peroxidation, cells were seeded in 96-well plates and treated with FAC and BSO as described in Materials and Methods. On day 4, $10 \mu \mathrm{M}$ Bodipy C11 581/591 was added and the cells were incubated for 1 hour. As a positive control for lipid peroxidation, cells seeded in parallel were treated with $100 \mathrm{nM}$ RSL-3 overnight; as a negative control, cells were treated with drug vehicle, DMSO. Lipid peroxidation was assessed as the ratio of $591_{\text {emission }} / 510_{\text {emission }}$ fluorescence (see Materials and Methods) and normalized by cell number. The experiments are representative of at least two independent experiments, and the data shown are the mean and S.D. values calculated from four replicates. $* * * P<0.001$ by two-sided $t$ test.

We used a small molecule, erastin, that specifically triggers this pathway, as well as small molecules that inhibit ferroptosis, such as Fer-1, SRS11-92, and XJB-5-131, as probes in cellular models of FRDA, focusing on cell lines that have been used to understand the biology of FRDA, and to test compounds with therapeutic potential. Patient-derived primary fibroblasts, although a clinically unaffected cell type, are sensitive to treatment with iron (Wong et al., 2000) and BSO (Jauslin et al., 2002), which inhibits glutathione synthesis. Based on these data, we developed a cellular model of FRDA in which primary FRDA fibroblasts were treated with a synergistic combination of FAC and $\mathrm{BSO}$, which we have used successfully to screen for potential therapeutics for FRDA (Cotticelli et al., 2012). We demonstrate herein that the ferroptosis inhibitors Fer-1, SRS11-92, and XJB-5-131 are efficacious in protecting FRDA cells in this model, whereas caspase inhibitors are not. The efficacy of XJB-5-131 is particularly relevant to the FRDA field since iron overload in FRDA cells occurs in the mitochondria, and XJB-5-131 is highly enriched in mitochondria, where it acts as a free electron, reactive-oxygen species, and radical scavenger, and prevents the oxidation of the mitochondrial membrane lipid cardiolipin (Kagan et al., 2009). XJB-5-131 has also already shown efficacy in vivo in rodent models of neurodegeneration. Fer-1 and SRS11-92 have previously been found to slow the accumulation of lipid hydroperoxides, possibly due to inhibition of lipoxygenases, but more likely derived from the radical trapping properties of these antioxidants (Zilka et al., 2017).

In the present study, we also show that lipid peroxidation increases in cells treated with iron and BSO and that GPX activity decreases, confirming and expanding on our previous finding that deuterated polyunsaturated fatty acids are sufficient to rescue cell viability (Cotticelli et al., 2013). The critical role of lipid peroxidation in ferroptosis has also been demonstrated using the same deuterated polyunsaturated fatty acids by Yang et al. (2016). Taken together, these data indicate that FRDA cells treated with FAC and BSO activate a ferroptotic pathway, and that treatment of FRDA cells with FAC and BSO is a useful model for screening ferroptosis inhibitors. The model also recapitulates some critical features of FRDA, and compounds we have previously tested in this model have gone on to further testing in patients, among them a deuterated polyunsaturated fatty acid that is now in a phase I/II clinical trial for FRDA (Zesiewicz et al., 2018). Indirect evidence of ferroptosis can be seen even in cells not challenged with oxidative stress. We demonstrate herein that human and murine FRDA fibroblasts are more sensitive to erastin, including cells derived from KIKO mice, a wellcharacterized mouse model of the disorder. Knocking down 
B

A

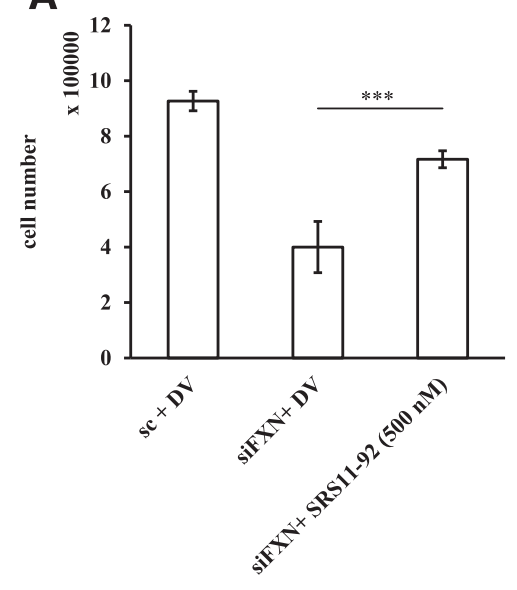

C
$48 \mathrm{~h}$
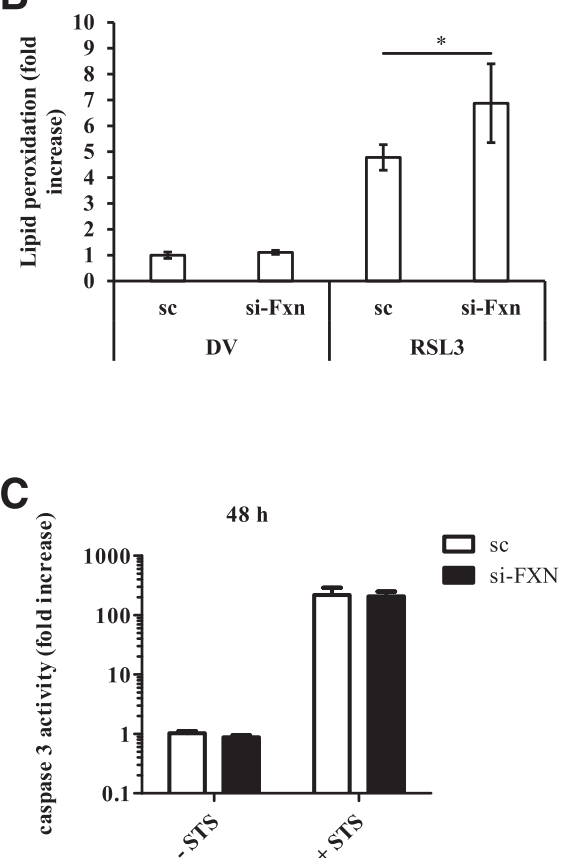
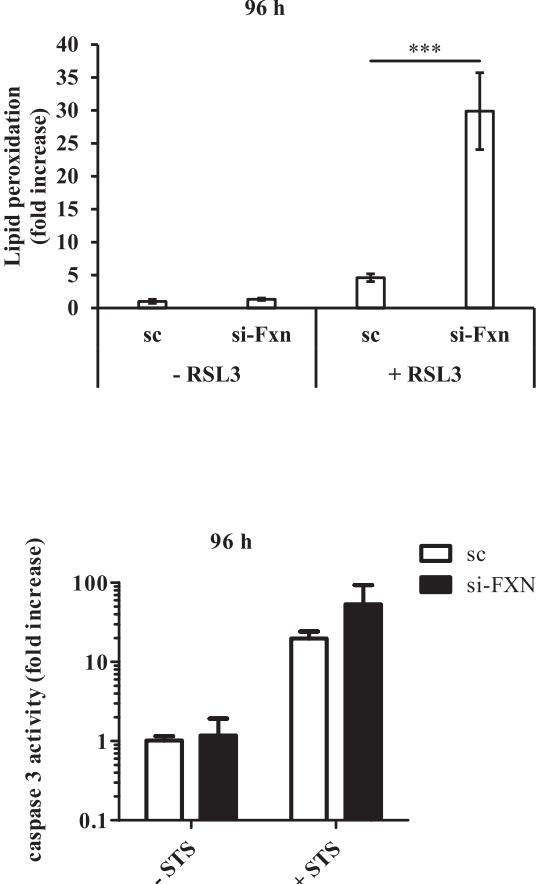

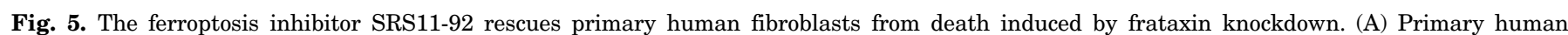

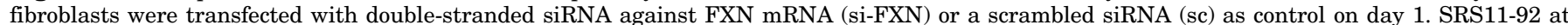

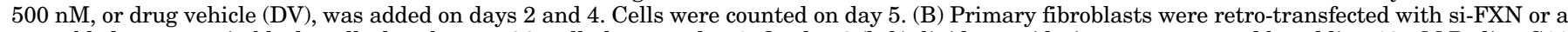

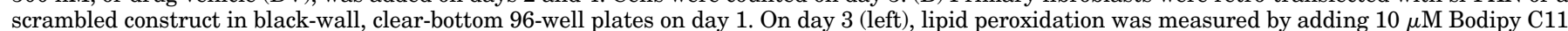

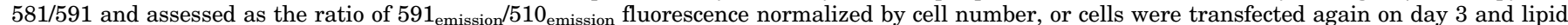

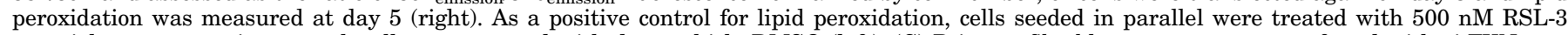

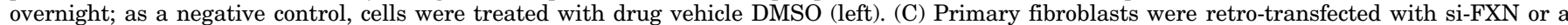

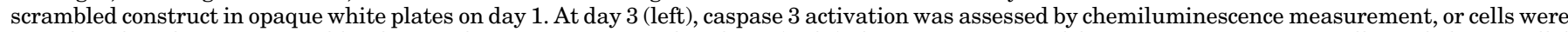

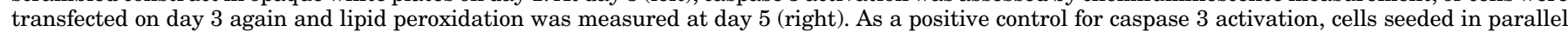

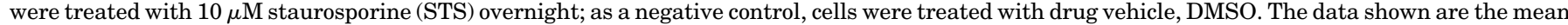
and S.D. values calculated from four replicates. $* P<0.05$ by two-sided $t$ test; $* * * P<0.001$ by two-sided $t$ test.

frataxin is also sufficient to induce cell death, which can be partially rescued by SRS11-92. In this latter model knocking down frataxin leads to an increase in lipid peroxidation that can be measured indirectly as increased sensitivity to RSL3, a ferroptosis inducer. Under the same conditions, caspase 3 activation, measured as increased sensitivity to staurosporine, cannot be detected unless frataxin levels are further decreased. Taken together, our data suggest that in the models we have studied the threshold for ferroptosis activation due to low frataxin is lower than that for apoptosis.

A limitation of the present study is the fact that although FRDA fibroblasts exhibit many of the hallmarks of FRDA pathophysiology, including mitochondrial iron overload, mitochondrial dysfunction, and increased sensitivity to oxidative stress, fibroblasts are not an affected cell type in FRDA in vivo. In FRDA, there is a slow and progressive neurodegeneration and a replacement of cardiomyocytes with fibrotic tissue in those patients that develop cardiomyopathy. Therefore, it will be important to determine whether ferroptosis contributes in vivo to one or both processes. Our data support further studies in this direction. Another limitation in modeling FRDA in vitro, in a system that is amenable to drug discovery, relates to the intrinsic nature of the disorder: disease progression, although irreversible, is slow, which allows the cells to compensate for low frataxin levels and reach a steady state in which they are far from healthy but still viable. The models described in this study involve loss of viability over several days. The efficacy of the compounds we tested-in both the
FAC/BSO model as well as the knockdown model-is time dependent, suggesting that there might be a window of opportunity during which ferroptosis inhibitors could be therapeutically beneficial in FRDA, perhaps by raising the threshold for ferroptotic cell death and thereby buying time for cells to repair damaged lipids (for example) before the threshold is reached.

\section{Acknowledgments}

We thank our colleagues at the Center of Excellence for Friedreich's Ataxia Research for helpful discussions and David Lynch (Children's Hospital of Philadelphia) for the primary human DL6030 fibroblasts.

\section{Authorship Contributions}

Participated in research design: Cotticelli, Xia, Wilson.

Conducted experiments: Cotticelli, Xia, Lee, Lin.

Contributed new reagents or analytic tools: Wipf, Huryn, Terrab. Performed data analysis: Cotticelli, Wilson.

Wrote or contributed to the writing of the manuscript: Cotticelli, Wilson.

\section{References}

Armstrong JS, Khdour O, and Hecht SM (2010) Does oxidative stress contribute to the pathology of Friedreich's ataxia? A radical question. FASEB $J$ 24:2152-2163. Belmokhtar CA, Hillion J, and Ségal-Bendirdjian E (2001) Staurosporine induces apoptosis through both caspase-dependent and caspase-independent mechanisms. Oncogene 20:3354-3362.

Bradley JL, Blake JC, Chamberlain S, Thomas PK, Cooper JM, and Schapira AH (2000) Clinical, biochemical and molecular genetic correlations in Friedreich's ataxia. Hum Mol Genet 9:275-282.

Calmels N, Schmucker S, Wattenhofer-Donzé M, Martelli A, Vaucamps N, Reutenauer L, Messaddeq N, Bouton C, Koenig M, and Puccio H (2009) The first cellular models based on frataxin missense mutations that reproduce spontaneously the defects associated with Friedreich ataxia. PLoS One 4:e6379. 
Collins A (2013) Clinical neurogenetics: Friedreich ataxia. Neurol Clin 31:1095-1120. Cotticelli MG, Crabbe AM, Wilson RB, and Shchepinov MS (2013) Insights into the role of oxidative stress in the pathology of Friedreich ataxia using peroxidation resistant polyunsaturated fatty acids. Redox Biol 1:398-404.

Cotticelli MG, Rasmussen L, Kushner NL, McKellip S, Sosa MI, Manouvakhova A, Feng S, White EL, Maddry JA, Heemskerk J, et al. (2012) Primary and secondary drug screening assays for Friedreich ataxia. J Biomol Screen 17:303-313.

Cotticelli MG, Xia S, Kaur A, Lin D, Wang Y, Ruff E, Tobias JW, and Wilson RB (2018) Identification of p38 MAPK as a novel therapeutic target for Friedreich's ataxia. Sci Rep 8:5007.

Delatycki MB, Camakaris J, Brooks H, Evans-Whipp T, Thorburn DR, Williamson R, and Forrest SM (1999) Direct evidence that mitochondrial iron accumulation occurs in Friedreich ataxia. Ann Neurol 45:673-675.

Dixon SJ, Lemberg KM, Lamprecht MR, Skouta R, Zaitsev EM, Gleason CE, Patel DN, Bauer AJ, Cantley AM, Yang WS, et al. (2012) Ferroptosis: an iron-dependent form of nonapoptotic cell death. Cell 149:1060-1072.

Gomes CM and Santos R (2013) Neurodegeneration in Friedreich's ataxia: from defective frataxin to oxidative stress. Oxid Med Cell Longev 2013:487534.

Jauslin ML, Wirth T, Meier T, and Schoumacher F (2002) A cellular model for Friedreich ataxia reveals small-molecule glutathione peroxidase mimetics as novel treatment strategy. Hum Mol Genet 11:3055-3063.

Kagan VE, Wipf P, Stoyanovsky D, Greenberger JS, Borisenko G, Belikova NA, Yanamala N, Samhan Arias AK, Tungekar MA, Jiang J, et al. (2009) Mitochondria targeting of electron scavenging antioxidants: regulation of selective oxidation vs random chain reactions. Adv Drug Deliv Rev 61:1375-1385.

Koeppen AH and Mazurkiewicz JE (2013) Friedreich ataxia: neuropathology revised. $J$ Neuropathol Exp Neurol 72:78-90.

Lin H, Magrane J, Rattelle A, Stepanova A, Galkin A, Clark EM, Dong YN, Halawani SM, and Lynch DR (2017) Early cerebellar deficits in mitochondrial biogenesis and respiratory chain complexes in the KIKO mouse model of Friedreich ataxia. Dis Model Mech 10:1343-1352.

Lodi R, Hart PE, Rajagopalan B, Taylor DJ, Crilley JG, Bradley JL, Blamire AM, Manners D, Styles P, Schapira AHV, et al. (2001) Antioxidant treatment improves in vivo cardiac and skeletal muscle bioenergetics in patients with Friedreich's ataxia. Ann Neurol 49:590-596.

Miranda CJ, Santos MM, Ohshima K, Smith J, Li L, Bunting M, Cossée M, Koenig M, Sequeiros J, Kaplan J, et al. (2002) Frataxin knockin mouse. FEBS Lett 512 291-297.
Rötig A, de Lonlay P, Chretien D, Foury F, Koenig M, Sidi D, Munnich A, and Rustin $\mathrm{P}$ (1997) Aconitase and mitochondrial iron-sulphur protein deficiency in Friedreich ataxia. Nat Genet 17:215-217.

Seluanov A, Vaidya A, and Gorbunova V (2010) Establishing primary adult fibroblast cultures from rodents. $J$ Vis Exp 44:2033.

Skouta R, Dixon SJ, Wang J, Dunn DE, Orman M, Shimada K, Rosenberg PA, Lo DC, Weinberg JM, Linkermann A, et al. (2014) Ferrostatins inhibit oxidative lipid damage and cell death in diverse disease models. J Am Chem Soc 136: $4551-4556$

Stockwell BR, Friedmann Angeli JP, Bayir H, Bush AI, Conrad M, Dixon SJ, Fulda S Gascón S, Hatzios SK, Kagan VE, et al. (2017) Ferroptosis: a regulated cell death nexus linking metabolism, redox biology, and disease. Cell 171:273-285.

Wipf P, Xiao J, Jiang J, Belikova NA, Tyurin VA, Fink MP, and Kagan VE (2005) Mitochondrial targeting of selective electron scavengers: synthesis and biological analysis of hemigramicidin-TEMPO conjugates. J Am Chem Soc 127:12460-12461.

Wong A, Yang J, Cavadini P, Gellera C, Lonnerdal B, Taroni F, and Cortopassi G (1999) The Friedreich's ataxia mutation confers cellular sensitivity to oxidant stress which is rescued by chelators of iron and calcium and inhibitors of apoptosis. Hum Mol Genet 8:425-430.

Wong A, Yang J, Danielson S, Gellera C, Taroni F, and Cortopassi G (2000) Sensitivity of FRDA lymphoblasts to salts of transition metal ions. Antioxid Redox Signal 2:461-465.

Yang WS, Kim KJ, Gaschler MM, Patel M, Shchepinov MS, and Stockwell BR (2016) Peroxidation of polyunsaturated fatty acids by lipoxygenases drives ferroptosis. Proc Natl Acad Sci USA 113:E4966-E4975.

Zesiewicz T, Heerinckx F, De Jager R, Omidvar O, Kilpatrick M, Shaw J, and Shchepinov MS (2018) Randomized, clinical trial of RT001: early signals of efficacy in Friedreich's ataxia. Mov Disord 33:1000-1005.

Zilka O, Shah R, Li B, Friedmann Angeli JP, Griesser M, Conrad M, and Pratt DA (2017) On the mechanism of cytoprotection by ferrostatin-1 and liproxstatin-1 and the role of lipid peroxidation in ferroptotic cell death. ACS Cent Sci 3:232-243.

Address correspondence to: Robert B. Wilson, Department of Pathology and Laboratory Medicine, Children's Hospital of Philadelphia, Room 6024, Colket Translational Research Building, 3501 Civic Center Blvd., Philadelphia, PA 19104. E-mail: wilsonr@pennmedicine.upenn.edu 